\title{
Simple Analytic Expressions for the Total Born Approximation Cross Section for Pair Production in a Coulomb Field
}

\author{
L. C. Maximon \\ Institute for Basic Standards, National Bureau of Standards, Washingion, D.C. 20234
}

(January 9, 1968)

\begin{abstract}
The total Born approximation cross section for pair production in a coulomb field given originally by Racah is used to derive two simple and rapidly convergent analytic expansions for this cross section, one valid for high energies, $k$, of the incident photon, the other for energies near the threshold. For $k>4 m c^{2}$ the fractional error involved in using the first four terms in the high energy expansion is $<4.4 \times 10^{-5}$. For $k<4 m c^{2}$ the fractional error committed in using the first five terms of the low energy expansion is $<1.1 \times 10^{-4}$. The leading terms in these expansions are the well known high energy and low energy limits of this cross section, respectively.
\end{abstract}

Key Words: Born approximation, coulomb field, moderate energy cross section, pair production, Racah cross section, total photon cross section.

The cross section for the production of an electron-positron pair in the field of an unscreened Coulomb potential $V=-Z e^{2} / r$ was first given in first Born approximation in a number of papers nearly thirty five years ago by W. Heitler and F. Sauter [1], ${ }^{1}$ H. Bethe and W. Heitler [2, 3], and G. Racah [4]. References 1, 2, and 3 give this cross section, differential in energy but integrated over all particle directions, without any approximation in the energy [5] as well as in a form valid when the energy of each of the pair particles is extremely relativistic (E.R.) [6], $\left(\epsilon_{1} \gg 1, \epsilon_{2}>1\right)$ :

where

$$
\frac{d \sigma}{d \epsilon_{1}}=4 \bar{\phi} \frac{\epsilon_{1}^{2}+\epsilon_{2}^{2}+\frac{2}{3} \epsilon_{1} \epsilon_{2}}{k^{3}}\left(\ln \frac{2 \epsilon_{1} \epsilon_{2}}{k}-\frac{1}{2}\right)
$$

$$
\bar{\phi}=\frac{e^{2}}{\hbar c}\left(\frac{Z e^{2}}{\mathrm{mc}^{2}}\right)^{2}=Z^{2} \cdot 5.7938 \times 10^{-28} \mathrm{~cm}^{2} .
$$

and $\epsilon_{1}, \epsilon_{2}, k$ are the energies of the positron, electron and photon respectively, in units of $m c^{2}$. References 2 and 3 give the total cross section (eq (1) integrated over $\epsilon_{1}$ ), but only in the extreme relativistic limit $(k \gg 1)$ :

$$
\sigma=\bar{\phi}\left(\frac{28}{9} \ln 2 k-\frac{218}{27}\right) . \quad \text { (E.R.) }
$$

Only in the papers by Racah can one find an expression for the total cross section involving no high energy approximations. Unfortunately, this expression involves indefinite integrals over elliptic integrals, so that it is quite inconvenient for obtaining numerical values of the total cross section. In fact, for this purpose it is equally simple to integrate directly, numerically, the differential cross section [5] in which no high energy approximations have been made. However, both

' Figures in brackets indicate the literature references at the end of this paper. 
for numerical evaluation and for an understanding of the analytical approximations involved in the extreme relativistic expression (3), it is clear that it would be convenient to have a simple analytic expression for the total cross section, valid over a large range of energies, which reduces in an obvious manner to (3) in the limit of large $k$. A step in this direction was made in a paper by Hough [7] who gives a corrected expression for the total cross section:

$$
\sigma=\bar{\phi}\left(\frac{28}{9} \ln 2 k-\frac{218}{27}-\frac{6.45}{k}\right)
$$

to be applied at lower energies than those for which (3) may be used. In addition, a number of empirical analytical expressions are given to cover the region $2 \leqslant k \leqslant 15$. They are, however, not really very simple, and they do not go smoothly into the high energy expression. Indeed, while the expression (4) is, as far as numerical values are concerned, an improvement over (3) for moderate energies, it is rather deceptive from the analytical standpoint: The corrections to (3) for moderate $k$ are not of the form $1 / k$. The expression (4) results in fact from an integration of the high energy differential cross section (1) between the limits $\epsilon=1$ and $\epsilon_{1}=k-1$ rather than 0 and $k$, which leads to (3):

$$
\sigma=\frac{4 \bar{\phi}}{k^{3}} \int_{0}^{k}\left(\epsilon_{1}^{2}+\epsilon_{2}^{2}+\frac{2}{3} \epsilon_{1} \epsilon_{2}\right)\left(\ln \frac{2 \epsilon_{1} \epsilon_{2}}{k}-\frac{1}{2}\right) d \epsilon_{1}=\bar{\phi}\left(\frac{28}{9} \ln 2 k-\frac{218}{27}\right),
$$

whereas

$\sigma=\frac{4 \bar{\phi}}{k^{3}} \int_{1}^{k-1}\left(\epsilon_{1}^{2}+\epsilon_{2}^{2}+\frac{2}{3} \epsilon_{1} \epsilon_{2}\right)\left(\ln \frac{2 \epsilon_{1} \epsilon_{2}}{k}-\frac{1}{2}\right) d \epsilon_{1}=\bar{\phi}\left(\frac{28}{9} \ln 2 k-\frac{218}{27}+\frac{12-8 \ln 2}{k}\right)\left(1+\mathrm{O}\left(\frac{1}{k^{2}}\right)\right)$

in which $12-8 \ln 2=6.45 \ldots$

However, the extreme relativistic differential cross section in the integrands here has errors of relative order $1 / \epsilon_{1}^{2}$ and $1 / \epsilon_{2}^{2}$, and these may be expected to give contributions of order

$$
\frac{1}{k^{3}} \int_{1}^{k-1} \frac{\left(\epsilon_{1}^{2}+\epsilon_{2}^{2}+\frac{2}{3} \epsilon_{1} \epsilon_{2}\right)}{\epsilon_{1}^{2}}\left(\ln \frac{2 \epsilon_{1} \epsilon_{2}}{k}-\frac{1}{2}\right) d \epsilon_{1}=\mathrm{O}\left(\frac{\ln k}{k}\right), \mathrm{O}\left(\frac{1}{k}\right),
$$

i.e., of exactly the same order as the correction calculated by changing the limits of integration from $(0, k)$ to $(1, k-1)$. This is in fact borne out by the expansion given here, in which we see that no terms of the form $1 / k$ actually appear in successive terms in the high energy expansion.

In this note we return therefore to the expression for the total cross section without any approximation in the energy, given by Racah, and derive from it two relatively simple and rapidly convergent expansions - one for large $k$, the other for $k$ near threshold $(k=2)$. The first few terms in each of these expansions are shown to suffice for both analytic and numerical purposes for all $k$.

The results which we obtain are the following: For $k$ near threshold we derive in fact two series, one in the expansion parameter

$$
\eta=\frac{k-2}{k+2}
$$

the other in the somewhat more complicated expansion parameter

$$
\rho=\frac{2 \eta}{1+\sqrt{1-\eta^{2}}}
$$


This second series has the advantage of being more rapidly convergent. These expansions are

$$
\sigma=\bar{\phi} \frac{2 \pi}{3}\left(\frac{k-2}{k}\right)^{3}\left[1+\frac{1}{2} \eta+\frac{23}{40} \eta^{2}+\frac{37}{120} \eta^{3}+\frac{61}{192} \eta^{4}+\ldots .\right]
$$

and

$$
\sigma=\bar{\phi} \frac{2 \pi}{3}\left(\frac{k-2}{k}\right)^{3}\left[1+\frac{1}{2} \rho+\frac{23}{40} \rho^{2}+\frac{11}{60} \rho^{3}+\frac{29}{960} \rho^{4}+\ldots\right]
$$

For large $k$ we derive an expansion in the parameter

$$
\begin{aligned}
\mu^{2}=\left(\frac{2}{k}\right)^{2}: & \\
\sigma= & \bar{\phi}\left\{\frac{28}{9} \ln 2 k-\frac{218}{27}\right. \\
& +\left(\frac{2}{k}\right)^{2}\left[6 \ln 2 k-\frac{7}{2}+\frac{2}{3} \ln ^{3} 2 k-\ln ^{2} 2 k-\frac{1}{3} \pi^{2} \ln 2 k+2 \zeta(3)+\frac{\pi^{2}}{6}\right] \\
& -\left(\frac{2}{k}\right)^{4}\left[\frac{3}{16} \ln 2 k+\frac{1}{8}\right] \\
& \left.-\left(\frac{2}{k}\right)^{6}\left[\frac{29}{9 \cdot 256} \ln 2 k-\frac{77}{27 \cdot 512}\right]+. .\right\}
\end{aligned}
$$

where

$$
\zeta(3)=\sum_{n=1}^{\infty} \frac{1}{n^{3}}=1.2020569 \ldots
$$

The convergence of this high energy expansion is extremely rapid, even for rather low energies. For example, for $k=4$, the fractional error, $\delta,\left(\sigma_{\text {approx }}=\sigma_{\text {exact }}(1+\delta)\right)$ involved in using the terms shown explicitly in (12) is only $+4.4 \times 10^{-5}$. The fractional errors obtained using the terms shown explicitly in (9) and (10), also for $k=4$, are $-1.1 \times 10^{-3}$ and $-1.1 \times 10^{-4}$, respectively. For $k>4$, the errors in (12) become smaller and for $k<4$ the errors in (9) and (10) become smaller.

The first term in each of these expansions has been given previously by Racah [8]. Their regions of validity are shown in figure 1, where we give graphs of the ratio of the first term in (10) $\left(\sigma_{1}^{\text {threshold }}=\bar{\phi} \frac{2 \pi}{3}\left(\frac{k-2}{k}\right)^{3}\right)$, and of the first term in (12) $\left(\sigma_{1}^{\text {E.R. }}=\bar{\phi}\left(\frac{28}{9} \ln 2 k-\frac{218}{27}\right)\right)$, to the exact total cross section, $\sigma$. It is to be noted from figure 1 that the first term of (10), valid for $k$ near threshold, is more than 10 percent off for $k>1.5 \mathrm{MeV}$, and that the first term of (12), valid for $k$ very large, is more than 10 percent off for $k<10 \mathrm{MeV}$. In figure 2 we give a graph of the total cross section, $\sigma$, in millibarns, as a function of the incident photon energy, $k$, in MeV. In table 1, values, $(\sigma / \bar{\phi})$, of the total cross section in units of $\bar{\phi}$ are given for photon energies ranging from 1.03 to $1000 \mathrm{MeV}$.

We proceed now to the derivation of these expansions, our starting point being the exact Born approximation cross section as given by Racah:

$$
\begin{aligned}
\sigma=\bar{\phi}\left\{\frac{692+468 \eta+76 \eta^{2}+108 \eta^{2}}{27(1+\eta)^{3}} K(\eta)\right. & -\frac{692+360 \eta+692 \eta^{2}}{27(1+\eta)^{3}} E(\eta) \\
& \left.-4 \frac{(1-\eta)^{2}}{(1+\eta)^{2}}\left[\int_{0}^{\eta} \frac{K(\xi)}{1-\xi} d \xi-4 \int_{0}^{\eta} \frac{d \zeta}{1-\zeta^{2}} \int_{0}^{\zeta} \frac{K(\xi)}{1-\xi} d \xi\right]\right\}
\end{aligned}
$$




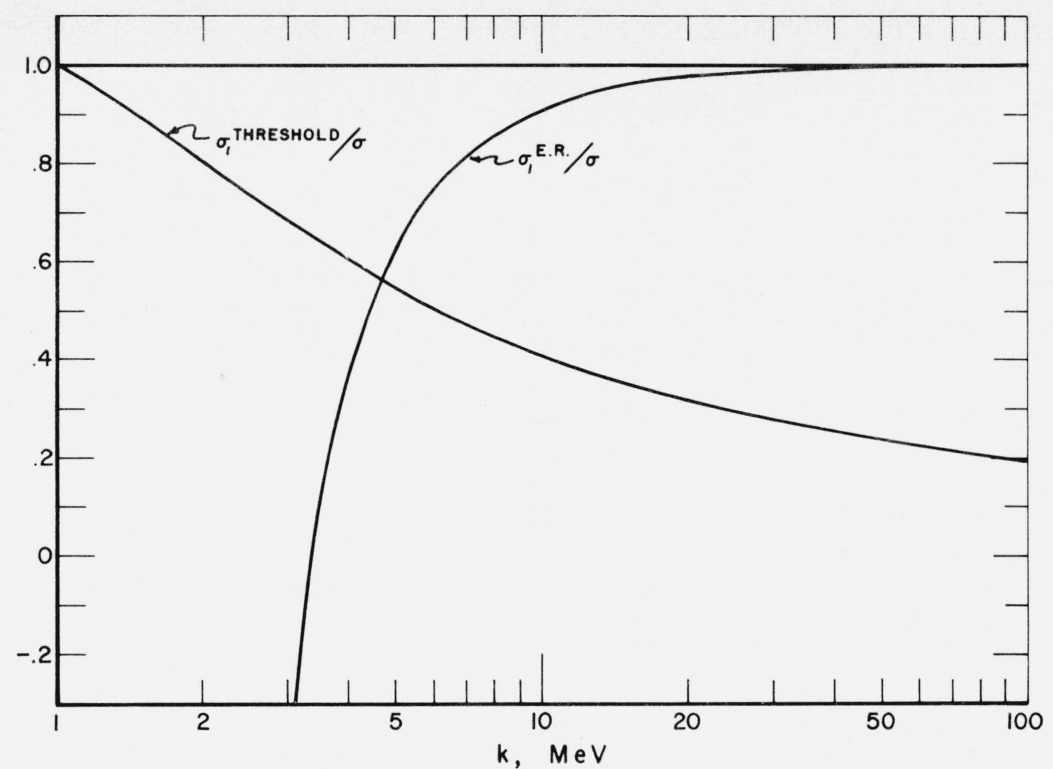

FIGURE 1. The ratio of the first term in expansion (10) $\left(\sigma_{1}^{\text {threshold }}=\bar{\phi} \cdot \frac{2 \pi}{3}\left(\frac{\mathrm{k}-2}{\mathrm{k}}\right)^{3}\right)$, and of the first term in expansion (12) $\left(\sigma_{1}^{E \cdot R .}=\bar{\phi}\left(\frac{28}{9} \ln 2 \mathrm{k}-\frac{218}{27}\right)\right)$, to the exact total cross section, $\sigma$, as a function of the incident photon energy $\mathrm{k}$, in $\mathrm{MeV}$.

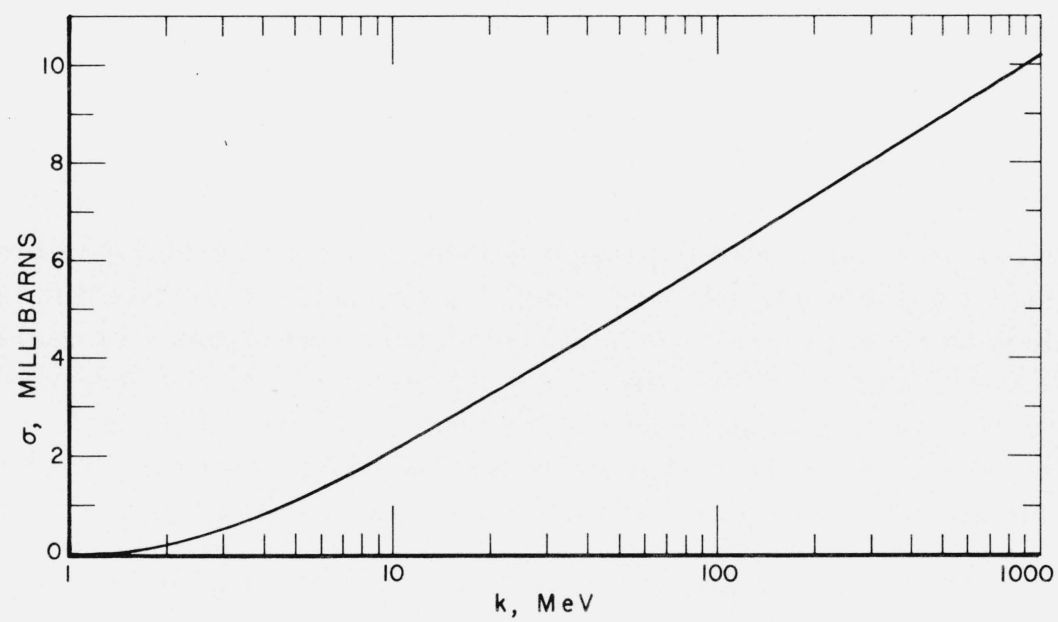

FIGURE 2. The total pair production cross section, $\sigma$, in millibarns, as a function of the incident photon energy, $\mathrm{k}$, in $\mathrm{MeV}$.

where $K(\eta)$ and $E(\eta)$ are the complete elliptic integrals of the first and second kind, respectively [9], and are defined by

$$
\begin{aligned}
K(\eta) & =\int_{0}^{1} \frac{d t}{\sqrt{\left(1-t^{2}\right)\left(1-\eta^{2} t^{2}\right)}} \\
& =\frac{\pi}{2} F\left(\frac{1}{2}, \frac{1}{2} ; 1 ; \eta^{2}\right)=\frac{\pi}{2} \sum_{n=0}^{\infty} \frac{\left(\frac{1}{2}\right)_{n}\left(\frac{1}{2}\right)_{n}}{n ! n !} \eta^{2 n}
\end{aligned}
$$


TABLE 1. The total cross section in units of $\bar{\phi}$ as a function of incident photon energy in $\mathrm{MeV}$

\begin{tabular}{l|c||r|r}
\hline \hline$k(\mathrm{MeV})$ & $\sigma / \bar{\phi}$ & $k(\mathrm{MeV})$ & $\sigma / \bar{\phi}$ \\
& & & \\
1.03 & $9.788 \times 10^{-7}$ & 10 & 3.647 \\
1.05 & $3.994 \times 10^{-5}$ & 15 & 4.782 \\
1.10 & $7.607 \times 10^{-4}$ & 20 & 5.618 \\
1.15 & $2.978 \times 10^{-3}$ & 30 & 6.826 \\
1.20 & $7.134 \times 10^{-3}$ & 40 & 7.696 \\
1.25 & $1.342 \times 10^{-2}$ & 50 & 8.377 \\
1.30 & $2.189 \times 10^{-2}$ & 60 & 8.936 \\
1.40 & $4.507 \times 10^{-2}$ & 80 & 9.822 \\
1.50 & $7.577 \times 10^{-2}$ & 100 & 10.511 \\
1.75 & $1.777 \times 10^{-1}$ & 150 & 11.767 \\
2.00 & $3.030 \times 10^{-1}$ & 200 & 12.659 \\
2.50 & $5.836 \times 10^{-1}$ & 300 & 13.919 \\
3.00 & $8.716 \times 10^{-1}$ & 400 & 14.813 \\
4.00 & 1.416 & 500 & 15.507 \\
5.00 & 1.900 & 600 & 16.074 \\
6.00 & 2.328 & 800 & 16.968 \\
8.00 & 3.052 & 1000 & 17.662 \\
& & & \\
\hline
\end{tabular}

$$
\begin{aligned}
E(\eta) & =\int_{0}^{1} \frac{\sqrt{1-\eta^{2} t^{2}}}{\sqrt{1-t^{2}}} d t \\
& =\frac{\pi}{2} F\left(-\frac{1}{2}, \frac{1}{2} ; 1 ; \eta^{2}\right)=\frac{\pi}{2} \sum_{n=0}^{\infty} \frac{\left(-\frac{1}{2}\right)_{n}\left(\frac{1}{2}\right)_{n}}{n ! n !} \eta^{2 n}
\end{aligned}
$$

where

$$
(a)_{n}=a(a+1) \ldots(a+n-1) \text { for } n>0
$$

and

$$
(a)_{0}=1 \text {. }
$$

Thus for $\eta<1$, we may write out the power series for $K$ and $E$ in (13), integrate term by term, and collect successive powers of $\eta$. This straightforward procedure leads to our expansion (9).

Expansion (10) is obtained by using the transformations [9]

$$
\begin{gathered}
K(\eta)=(1+\lambda) K(\lambda)=\frac{\pi}{2}(1+\lambda) F\left(\frac{1}{2}, \frac{1}{2} ; 1 ; \lambda^{2}\right) \\
E(\eta)=\frac{2}{1+\lambda} E(\lambda)-(1-\lambda) K(\lambda)=\frac{\pi}{2(1+\lambda)} F\left(-\frac{1}{2},-\frac{1}{2} ; 1 ; \lambda^{2}\right) \\
\lambda=\frac{1-\sqrt{1-\eta^{2}}}{1+\sqrt{1-\eta^{2}}}, \quad \eta=\frac{2 \sqrt{\lambda}}{1+\lambda} .
\end{gathered}
$$

The series for $K(\lambda)$ and $E(\lambda)$ converge faster than those for $K(\eta)$ and $E(\eta)$ since $\lambda<\eta$. Substituting (16), (17) and (18) in (13), we write out the power series for $K(\lambda), E(\lambda)$ and $\eta$ (a series in $\lambda^{1 / 2}$ ), perform the integrations term by term with respect to $\lambda$, collect successive powers of $\lambda^{1 / 2}$ and write the series in terms of the variable $\rho=2 \lambda^{1 / 2}=2 \eta /\left[1+\sqrt{1-\eta^{2}}\right]$ since $\rho \rightarrow \eta$ as $\eta \rightarrow 0$. This gives the expansion (10). 
In order to obtain the high energy expansion (12), we note that $\eta \sim 1$ for $k \gg 1$. We therefore need expansions of the functions $K(\eta)$ and $E(\eta)$ about $\eta=1$. To this end we rewrite the transformations (16) and (17) with $\lambda$ and $\eta$ interchanged:

$$
\begin{gathered}
K(\eta)=\frac{1}{1+\eta} K\left(\frac{2 \sqrt{\eta}}{1+\eta}\right) \\
E(\eta)=\frac{1+\eta}{2} E\left(\frac{2 \sqrt{\eta}}{1+\eta}\right)+\frac{1-\eta}{2} K\left(\frac{2 \sqrt{\eta}}{1+\eta}\right) . \\
\frac{2 \sqrt{\eta}}{1+\eta}=\sqrt{1-\left(\frac{2}{k}\right)^{2}} \\
\mu=\frac{2}{k}, \quad \mu^{\prime}=\sqrt{1-\mu^{2}}
\end{gathered}
$$

and write (19) and (20) in the form

$$
\begin{aligned}
& K(\eta)=\frac{k+2}{2 k} K\left(\mu^{\prime}\right) \\
& E(\eta)=\frac{k}{k+2} E\left(\mu^{\prime}\right)+\frac{2}{k+2} K\left(\mu^{\prime}\right) .
\end{aligned}
$$

In (13), the terms preceding the integrals can now be rewritten conveniently in the form

$$
\begin{array}{r}
\frac{692+468 \eta+76 \eta^{2}+108 \eta^{3}}{27(1+\eta)^{3}} K(\eta)-\frac{692+360 \eta+692 \eta^{2}}{27(1+\eta)^{3}} E(\eta)=\left[\frac{56}{9}+\frac{536}{27 k}+\frac{928}{27 k^{2}}+\frac{64}{9 k^{3}}\right] K(\eta) \\
-\left[\frac{218}{27}+\frac{512}{27 k^{2}}\right]\left[1+\frac{2}{k}\right] E(\eta)=\frac{2}{27}\left[42+125 \mu^{2}+6 \mu^{4}\right] K\left(\mu^{\prime}\right)-\frac{2}{27}\left[109+64 \mu^{2}\right] E\left(\mu^{\prime}\right) .
\end{array}
$$

The desired expansions of $K$ and $E$ are

$$
\begin{gathered}
K\left(\mu^{\prime}\right)=\sum_{n=0}^{\infty} \frac{\left(\frac{1}{2}\right)_{n}\left(\frac{1}{2}\right)_{n}}{n ! n !}\left[\psi(n+1)-\psi\left(n+\frac{1}{2}\right)-\ln \mu\right] \mu^{2 n} \\
E\left(\mu^{\prime}\right)=1+\frac{1}{4} \sum_{n=0}^{\infty} \frac{\left(\frac{1}{2}\right)_{n}\left(\frac{3}{2}\right)_{n}}{n !(n+1) !}\left[\psi(n+2)+\psi(n+1)-\psi\left(n+\frac{3}{2}\right)-\psi\left(n+\frac{1}{2}\right)-2 \ln \mu\right] \mu^{2 n+2}
\end{gathered}
$$

where $\psi(z)=\Gamma^{\prime}(z) / \Gamma(z)$, and, in particular, [10]

$$
\begin{aligned}
\psi(n) & =-\gamma+\sum_{l=1}^{n-1} \frac{1}{l}, \quad n>0 \\
\psi\left(n+\frac{1}{2}\right) & =-\gamma-2 \ln 2+2 \sum_{l=0}^{n-1} \frac{1}{2 l+1}, \quad n>0 \\
\psi(0) & =-\gamma, \quad \psi\left(\frac{1}{2}\right)=-\gamma-2 \ln 2 \\
\gamma & =\text { Euler's constant }=0.5772 \ldots .
\end{aligned}
$$


From (28) we may write

where

$$
\begin{aligned}
& \psi(n+1)-\psi\left(n+\frac{1}{2}\right)=s_{n}+2 \ln 2 \\
& s_{n}=-2\left[1-\frac{1}{2}+\frac{1}{3}-+\ldots-\frac{1}{2 n}\right], \quad n>0 \\
& s_{0}=0 .
\end{aligned}
$$

Equations (26) and (27) are then written in the form

$$
\begin{gathered}
K\left(\mu^{\prime}\right)=\sum_{n=0}^{\infty} \frac{\left(\frac{1}{2}\right)_{n}\left(\frac{1}{2}\right)_{n}}{n ! n !}\left[s_{n}+\ln 2 k\right] \mu^{2 n} \\
E\left(\mu^{\prime}\right)=1+\frac{1}{4} \sum_{n=0}^{\infty} \frac{\left(\frac{1}{2}\right)_{n}\left(\frac{3}{2}\right)_{n}}{n !(n+1) !}\left[s_{n+1}+s_{n}+2 \ln 2 k\right] \mu^{2 n+2}
\end{gathered}
$$

Substitution of (30) and (31) in the right hand side of (25) gives the desired high energy expansion of the terms in (13) preceding the integrals. We note that the terms in this expansion are either of the form of a power series in $\mu^{2}=(2 / k)^{2}$ or $\ln 2 k$ times a power series in $\mu^{2}$.

We next consider the high energy expansion of the integrals in (13). Each of these integrals has the factor

$$
\left(\frac{1-\eta}{1+\eta}\right)^{2}=\left(\frac{2}{k}\right)^{2}=\mu^{2}
$$

which is precisely our expansion parameter. The first integral is

$$
I_{1}=\int_{0}^{n} \frac{K(\xi)}{1-\xi} d \xi
$$

Let

$$
\xi=\frac{x-2}{x+2}
$$

from which

$$
I_{1}=\int_{2}^{k} \frac{d x}{x+2} K(\xi)
$$

Now from (19) we have

$$
K(\eta)=\frac{1}{1+\xi} K\left(\frac{2 \sqrt{\xi}}{1+\xi}\right)=\frac{x+2}{2 x} K\left(\sqrt{1-\left(\frac{2}{x}\right)^{2}}\right)
$$

so that

$$
I_{1}=\frac{1}{2} \int_{2}^{k} \frac{d x}{x} K\left(\sqrt{1-\left(\frac{2}{x}\right)^{2}}\right)=\frac{1}{2} \int_{\frac{2}{k}}^{1} \frac{d z}{z} K\left(\sqrt{1-z^{2}}\right), \quad z=\frac{2}{x}
$$


Since we want an expansion of $I_{1}$ for $k \gg 1$, i.e., $2 / k \ll 1$, we separate that part of the integrand which becomes singular as $z \rightarrow 0$. From (30)

$$
K\left(\sqrt{1-z^{2}}\right)-\ln \frac{4}{z}=\mathrm{O}\left(z^{2} \ln z\right)
$$

as $z \rightarrow 0$. Thus we may write

$$
\begin{aligned}
I_{1}=\frac{1}{2} \int_{\frac{2}{k}}^{1} \frac{d z}{z} & {\left[K\left(\sqrt{1-z^{2}}\right)-\ln \frac{4}{z}\right]+\frac{1}{2} \int_{\frac{2}{k}}^{1} \frac{d z}{z} \ln \frac{4}{z} } \\
& =\frac{1}{2} \int_{0}^{1} \frac{d z}{z}\left[K\left(\sqrt{1-z^{2}}\right)-\ln \frac{4}{z}\right]+\frac{1}{4} \ln ^{2} 2 k-\ln ^{2} 2-\frac{1}{2} \int_{0}^{\frac{2}{k}} \frac{d z}{z}\left[K\left(\sqrt{1-z^{2}}\right)-\ln \frac{4}{z}\right] .
\end{aligned}
$$

The evaluation of the definite integral in (38) is given in the appendix. The result is

$$
\int_{0}^{1} \frac{d z}{z}\left[K\left(\sqrt{1-z^{2}}\right)-\ln \frac{4}{z}\right]=2 \ln ^{2} 2-\frac{1}{12} \pi^{2} .
$$

In the last integral in (38) we substitute (30), with $\mu$ replaced by $z, 2 k$ by $4 / z$, and integrate term by term. We then obtain

$$
I_{1}=\frac{1}{4} \ln ^{2} 2 k-\frac{1}{24} \pi^{2}-\frac{1}{4} \sum_{n=1}^{\infty} \frac{\left(\frac{1}{2}\right)_{n}\left(\frac{1}{2}\right)_{n}}{n ! n ! n}\left[S_{n}+\frac{1}{2 n}+\ln 2 k\right]\left(\frac{2}{k}\right)^{2 n} .
$$

Finally, we consider the second integral in (13):

$$
I_{2}=-4 \int_{0}^{\eta} \frac{d \zeta}{1-\zeta^{2}} \int_{0}^{\zeta} \frac{K(\xi)}{1-\xi} d \xi=2 \int_{0}^{\eta}\left[\ln \left(\frac{1+\xi}{1-\xi}\right)-\ln \left(\frac{1+\eta}{1-\eta}\right)\right] \frac{K(\xi)}{1-\xi} d \xi
$$

upon integrating by parts. Again we substitute (33) and (35), and obtain

$$
I_{2}=-2 I_{1} \ln \frac{k}{2}-\int_{\frac{2}{k}}^{1} \frac{d z}{z} \ln z K\left(\sqrt{1-z^{2}}\right) .
$$

As with $I_{1}$, we write

$$
\begin{aligned}
& \int_{\frac{2}{k}}^{1} \frac{d z}{z} \ln z K\left(\sqrt{1-z^{2}}\right)=\int_{\frac{2}{k}}^{1} \frac{d z}{z} \ln z\left[K\left(\sqrt{1-z^{2}}\right)-\ln \frac{4}{z}\right]+\int_{\frac{2}{k}}^{1} \frac{d z}{z} \ln z \ln \frac{4}{z} \\
& =\int_{0}^{1} \frac{d z}{z} \ln z\left[K\left(\sqrt{1-z^{2}}\right)-\ln \frac{4}{z}\right]+\frac{1}{3} \ln ^{3} \frac{2}{k}-\ln 2 \ln ^{2} \frac{2}{k}-\int_{0}^{\frac{2}{k}} \frac{d z}{z} \ln z\left[K\left(\sqrt{1-z^{2}}\right)-\ln \frac{4}{z}\right] .
\end{aligned}
$$

The evaluation of the first integral here is also given in the appendix. The result is

$$
\int_{0}^{1} \frac{d z}{z} \ln z\left[K\left(\sqrt{1-z^{2}}\right)-\ln \frac{4}{z}\right]=\frac{4}{3} \ln ^{3} 2-\frac{1}{6} \pi^{2} \ln 2-\frac{1}{2} \zeta(3) .
$$


In the last integral in (43) in we again substitute (30) and integrate term by term. Since these integrals appear in (13) only in the form of the sum $I_{1}+I_{2}$, we give this result:

$I_{1}+I_{2}=-\frac{1}{6} \ln ^{3} 2 k+\frac{1}{4} \ln ^{2} 2 \mathrm{k}+\frac{1}{12} \pi^{2} \ln 2 k-\frac{1}{24} \pi^{2}-\frac{1}{2} \zeta(3)$

$$
-\frac{1}{4} \sum_{n=1}^{\infty} \frac{\left(\frac{1}{2}\right)_{n}\left(\frac{1}{2}\right)_{n}(n+1)}{n ! n ! n^{2}}\left[\ln 2 k+s_{n}+\frac{n+2}{2 n(n+1)}\right]\left(\frac{2}{k}\right)^{2 n}
$$

\section{Appendix}

We evaluate here the definite integrals appearing in (38) and (43):

$$
\begin{aligned}
& \left.J_{1}=\int_{0}^{1} \frac{d z}{z}[K) \sqrt{1-z^{2}}-\ln \frac{4}{z}\right] \\
& J_{2}=\int_{0}^{1} \frac{d z}{z} \ln z\left[K\left(\sqrt{1-z^{2}}\right)-\ln \frac{4}{z}\right] .
\end{aligned}
$$

We define

$$
J(\alpha)=\int_{0}^{1}\left[K\left(\sqrt{1-z^{2}}\right)-\ln \frac{4}{z}\right] z^{-1+2 \alpha} d z
$$

with $\alpha \geqslant 0$, so that

$$
\begin{gathered}
J_{1}=J(0) \\
J_{2}=\frac{1}{2} J^{\prime}(0) .
\end{gathered}
$$

Substituting $t=1-z^{2}$ in (A.3) and using (14b), we have

$$
J(\alpha)=\frac{\pi}{4} \int_{0}^{1}(1-t)^{-1+\alpha} F\left(\frac{1}{2}, \frac{1}{2} ; 1 ; t\right) d t+\frac{1}{4} \int_{0}^{1}(1-t)^{-1+\alpha} \ln \frac{1-t}{16} d t .
$$

Here

$$
\begin{gathered}
\int_{0}^{1}(1-t)^{-1+\alpha} d t=\frac{1}{\alpha} \\
\int_{0}^{1}(1-t)^{-1+\alpha} \ln (1-t) d t=\frac{d}{d \alpha} \int_{0}^{1}(1-t)^{-1+\alpha} d t=-\frac{1}{\alpha^{2}}
\end{gathered}
$$

and [11]

$$
\int_{0}^{1}(1-t)^{-1+\alpha} F\left(\frac{1}{2}, \frac{1}{2} ; 1 ; t\right) d t=\frac{\Gamma(\alpha)}{\Gamma(\alpha+1)} F\left(\frac{1}{2}, \frac{1}{2} ; 1+\alpha ; 1\right)=\left[\frac{\Gamma(\alpha)}{\Gamma\left(\alpha+\frac{1}{2}\right)}\right]^{2}
$$

Thus

$$
J(\alpha)=\frac{\Gamma^{2}\left(\frac{1}{2}\right) \Gamma^{2}(\alpha+1) \div \Gamma^{2}\left(\alpha+\frac{1}{2}\right)-4 \alpha \Gamma^{2}\left(\alpha+\frac{1}{2}\right) \ln 2}{4 \alpha^{2} \Gamma^{2}\left(\alpha+\frac{1}{2}\right)} .
$$


We then expand this expression in a power series in $\alpha$, keeping, in the final result, terms linear in $\alpha$. We note

$$
\begin{aligned}
& \Gamma^{\prime}(z)=\psi(z) \Gamma(z) \\
& \Gamma^{\prime \prime}(z)=\psi^{\prime}(z) \Gamma(z)+\psi^{2}(z) \Gamma(z)
\end{aligned}
$$

and the following values for specific values of $z:[10]$

$$
\begin{aligned}
& \Gamma(1)=1, \quad \Gamma\left(\frac{1}{2}\right)=\pi^{1 / 2} \\
& \psi(1)=-\gamma, \quad \psi\left(\frac{1}{2}\right)=-\gamma-2 \ln 2 \\
& \psi^{\prime}(1)=\frac{\pi^{2}}{6}, \quad \psi^{\prime}\left(\frac{1}{2}\right)=\frac{\pi^{2}}{2} \\
& \psi^{\prime \prime}(1)=-2 \zeta(3), \quad \psi^{\prime \prime}\left(\frac{1}{2}\right)=-14 \zeta(3) \\
& \zeta(3)=\sum_{n=1}^{\infty} \frac{1}{n^{3}}=1.2020569 \ldots
\end{aligned}
$$

We find

$$
J(\alpha)=2 \ln ^{2} 2-\frac{1}{12} \pi^{2}+a\left[\frac{8}{3} \ln ^{3} 2-\frac{1}{3} \pi^{2} \ln 2-\zeta(3)\right]+\mathrm{O}\left(\alpha^{2}\right)
$$

from which $J_{1}$ and $J_{2}$ follow from (A.4) and (A.5).

We would like to express our thanks to J. H. Hubbell for performing the computer calculations involved in the preparation of figures 1 and 2 and table 1.

\section{References}

[1] W. Heitler and F. Sauter, Nature 132, 892 (1933).

[2] H. Bethe and W. Heitler, Proc. Roy. Soc. London, Ser. A 146, 83 (1934).

[3] W. Heitler, The Quantum Theory of Radiation, third edition, pp. 256-267. (Oxford University Press, London, 1954).

[4] Giulio Racah, Nuovo Cimento 11, 477 (1934) and 13, 66 (1936). In the latter article, note p. 69, Eqs (10), (11) and the footnote on p. 69, where a typographical error in Eq (10) of the former paper is corrected.

[5] Ref. 3, p. 258, Eq (8).

[6] Ref. 3, p. 258, Eq (9).

[7] P. V. C. Hough, Phys. Rev. 73, 266 (1948).

[8] In Ref. 4, Nuovo Cimento 11, note p. 480, Eq (12).

[9] W. Gröbner and N. Hofreiter, Integroltafel, Vol. II (Springer-Verlag, Vienna, 3rd ed. 1961). Note pp. 39, 40.

[10] W. Magnus, F. Oberhettinger, and R. P. Soni, Formulas and Theorems for the Special Functions of Mathematical Physics (Springer-Verlag, New York, Inc., 1966). Note, p. 15.

[11] Ref. 9, pp. 40 and 55.

(Paper 72B1-261) 